\title{
Mesenchymal stem cells for pre-vascularization of engineered tissues
}

\begin{abstract}
Engineered tissues with a thickness larger than $150 \mu \mathrm{m}$ require an embedded functional vascular network to support cell survival and integration with host vasculature after in vivo implantation. Mesenchymal stem cells (MSCs) can secrete trophic factors to promote angiogenesis and function as pericytes to stabilize endothelial cell engineered neo-capillary structures. Recently, studies have shown that co-culturing MSCs with endothelial cells (ECs) could develop a pre-capillary network in tissue scaffolds. To realize such an outcome, several factors need to be considered, including MSC source, cell seeding order, oxygen concentration, and extra cellular matrix features. The present mini review summarizes these crucial considerations and will provide beneficial references for successful development of functional pre-vascularized tissues.
\end{abstract}

Volume 4 Issue 2 - 2018

\author{
Dhavan Sharma, Juan Felipe Chica, and Feng \\ Zhao
}

Department of Biomedical Engineering, Michigan Technological University, USA

Correspondence: Feng Zhao, Department of Biomedica Engineering, Michigan Technological University, 400 Townsend Drive, Houghton, MI 4993I, USA, Tel 90648728 52, Fax 9064 8717 17, Email fengzhao@mtu.edu

\section{Introduction}

Recent advances in the field of stem cell biology and tissue engineering have revolutionized therapeutic approaches to treat various diseases, especially chronic wounds, bone diseases, cardiovascular complications, and neurodegenerative diseases. Different stem cell types have been investigated for designing appropriate therapeutic treatments. Among them, approaches involving embryonic and induced pluripotent stem cells (iPSCs) are ethically and socially controversial. In addition, these stem cell types, due to their high pluripotency, contain risks of teratoma formation. ${ }^{1,2}$ In the past decade, mesenchymal stem cells (MSCs) have attracted considerable attention due to their straightforward and less invasive isolation procedure as well as their multi-differentiation potential. MSCs can differentiate into various cell types including osteoblasts, chondrocytes, adipocytes, smooth muscle like cells, endothelial like cells and cardiomyocyte like cells. Moreover, being immunoprivileged, allogenic MSCs encounter minimal risk of immune rejection. They also secrete various trophic factors, which can promote cell survival and tissue regeneration., ${ }^{3,4}$ These promising capabilities have made MSCs potential candidate for construction of various tissue-engineered products. Nevertheless, engineered tissues with a thickness larger than $150 \mu \mathrm{m}$ require a functional micro vascular network to supply gases, nutrients, metabolic byproducts, and integrate with host vasculature after implantation. ${ }^{5}$ In the physiological capillary structure, endothelial cells (ECs) surround the vessel lumen. These ECs are themselves wrapped by pericytes, which stabilize the capillary structure. ${ }^{6}$ Numerous studies have confirmed that MSCs can function as pericytes. ${ }^{7,8}$ Consequently, in order to develop a capillary network in tissue scaffolds various research groups over past several years have investigated the outcome of MSC-EC co-cultures. ${ }^{9-12}$ Compared with other pericyte candidates, MSCs are expected to play dual roles: stabilizing engineered micro vessels and performing their stem cell functions after implantation. In this mini review, we discuss important considerations for successful MSC-EC co-cultures to achieve a robust vascular network. These considerations include an appropriate cell source, cell-seeding order, optimum oxygen $\left(\mathrm{O}_{2}\right)$ levels, appropriate extracellular matrix (ECM) and tissue scaffold features (Figure 1).

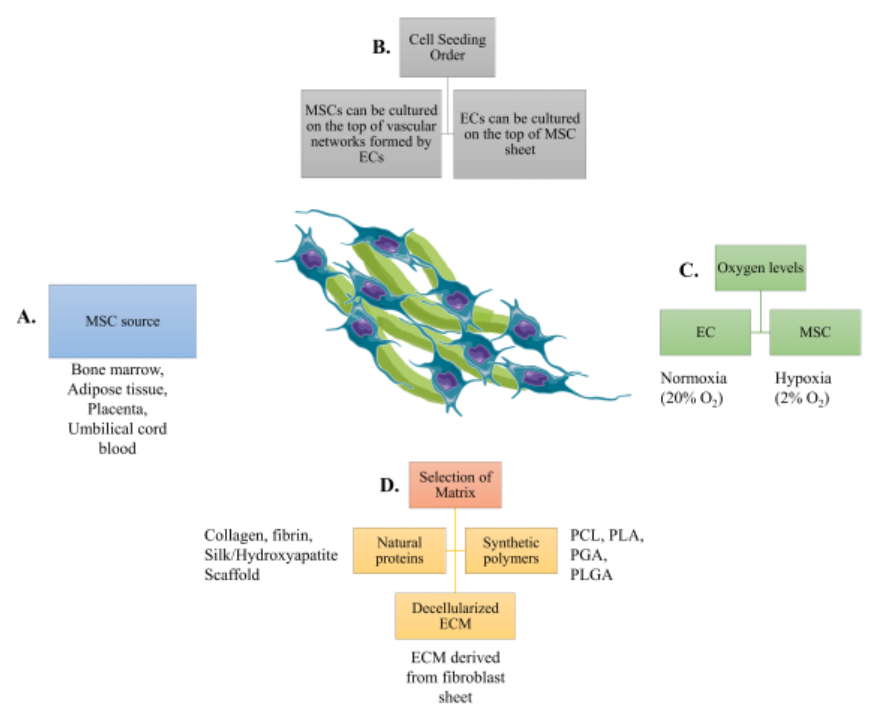

Figure I Considerations for MSC-EC co-culture for development of prevascularized engineered tissues. (A) Various sources from which MSCs can be isolated, (B) MSCs can be cultured on preformed vascular networks formed by ECs. In contrast, ECs cultured on MSC sheet forms better vascular networks, (C) MSCs maintain stemness and increase angiogenic growth factor secretion in a hypoxic environment. Whereas, ECs prefer normoxic environment for cell survival, proliferation and development of vascular networks, (D) Various natural and synthetic materials support MSC-EC co-culture. Decellularized ECM promotes development of robust vascular networks.

\section{Considerations for MSC-EC co-culture}

\section{MSC source}

MSCs can be isolated from many sites in the body including bone marrow, adipose tissue, placenta, umbilical cord and umbilical cord blood. ${ }^{13}$ Each of these tissues provides a unique microenvironment, which might affect MSCs' potential for vascular development during co-culture. Thus, it is crucial to compare MSCs derived from different tissue sources. Bone marrow derived MSCs are the most frequently 
used for biomedical applications. However, their isolation from bone marrow is associated with a low yield and high discomfort for patients. ${ }^{14}$ An alternative MSC source is adipose tissue, which is more accessible and provides a higher yield. ${ }^{15} \mathrm{Ma} \mathrm{J}$ et al. ${ }^{16}$ compared the angiogenic potential of bone marrow derived MSCs and adipose tissue derived MSCs by co-culturing them with human umbilical vein endothelial cells (HUVECs) ${ }^{16}$ Results from their animal study indicated that MSCs derived from these two sources have equal angiogenic potential. ${ }^{16}$ Besides bone marrow and adipose derived MSCs, placenta derived hMSCs showed a higher proliferation rate, multi-lineage differentiation capability and lower immunogenicity due to lack of human leukocyte antigen - antigen D Related (HLA-DR) gene fragment, which is a major histocompatibility complex (MHC) class II cell surface receptor. ${ }^{17}$ Similarly, umbilical cord blood derived MSCs have a high immunomodulatory effect due to lack of MHC-II expression. Thus, these MSCs can suppress the function of mature dendritic cells and proliferation of peripheral blood mononuclear cells. ${ }^{18}$ Despite these advantages, further studies are required to coculture placenta and cord blood derived MSCs with ECs, as they can be easily isolated from these sources without relying on painful invasive methods.

\section{Cell seeding order}

In addition to the cell source, the selection of appropriate cell seeding order into the scaffolds is another crucial consideration for MSC-EC co-culture. During physiological angiogenesis, ECs first proliferate to develop proangiogenic cord like structures and then recruit pericytes to stabilize the newly formed vasculature. ${ }^{6}$ Similarly, during an in vitro experimental setup on a collagen-glycosaminoglycan scaffold, it was discovered that a delayed addition of MSCs on preformed EC networks during co-culture promoted vascular network formation relative to simultaneous seeding. EC-MSC co-seeding created cellular aggregation on the scaffold surface, which resulted in poor cell infiltration and immature vasculature formation. ${ }^{19}$ Moreover, physiologically larger MSCs also interfered with early self-assembly of EC networks. MSCs secrete vascular endothelial growth factor (VEGF), a critical growth factor that promotes angiogenesis. Addition of MSCs after 3 days of EC monoculture promoted VEGF expression, which further enhanced vascular network density compared to simultaneous addition of both cell types. The pre-vascularized functional construct showed anastomosis between engineered vessels and host vasculature upon sub-subcutaneous implantation in a rat model. ${ }^{19}$ In another study, an opposite cell-seeding order was employed to develop pre-vascularized sheets by co-culturing ECs on the top of a thick MSC layer. ${ }^{9}$ This pre-vascularized sheet was subsequently folded to form a 3D construct, in which ECs migrated to form a vascular network in both horizontal and vertical directions. The engineered vascular network anastomosed with host vasculature after subcutaneous implantation in immunodeficient mice. ${ }^{9}$ Similarly, we have cultured ECs on top of MSC layers to form a high-density vascular network. ${ }^{20}$ When comparing capillary network formation using these two opposite cell seeding approaches, the EC seeding on top of MSC sheets develops a highly dense and mature vascular network.

\section{Oxygen levels}

During MSC culture, provision of an appropriate $\mathrm{O}_{2}$ level maintains stemness and trophic factor secretion. MSCs present in bone marrow are exposed to a hypoxic microenvironment with 1- $6 \% \mathrm{O}_{2}$ level, while vascular ECs experience higher $\mathrm{O}_{2}$ levels due to direct contact with $\mathrm{O}_{2}$ rich blood. ${ }^{21}$ To determine the effect of this physiological condition, we have examined the influence of physiological hypoxia $\left(2 \% \mathrm{O}_{2}\right)$ on MSC stemness maintenance and angiogenic growth factor secretion relative to a normoxic condition $\left(20 \% \mathrm{O}_{2}\right) .22$ Our results validated the beneficial effect of hypoxic preconditioning on hMSC's ability to form an improved vasculature compared to normoxic preconditioning. However, the subsequent coculture with ECs showed an opposite effect. The ECs cultured on top of the MSC sheet in a normoxic environment developed a longer, more extensively branched and uniform micro vascular network compared to their hypoxic counterpart. ${ }^{22}$ As mentioned above, ECs are generally exposed to higher $\mathrm{O}_{2}$ levels due to direct contact with blood. Thus, hypoxia might reduce their functional ability to construct capillary networks. We further implanted the prevascularized cell sheets, with combination of a split thickness skin graft, in a rat full thickness wound model. Results demonstrated that pre-vascularization of the MSC sheet significantly improved skin wound regeneration by alleviating graft contraction, preserving skin appendages, and inducing early neovascularization. ${ }^{20}$ These studies highlight the significance of $\mathrm{O}_{2}$ level during MSC and EC culture on the morphogenesis and function of engineered capillaries.

\section{Selection of appropriate matrix or scaffold}

Components of the ECM interact with cells and significantly affect their behavior. During early sprouting stages of angiogenesis in tissues, ECs break the underlying basal lamina and migrate toward connective tissue. Connective tissue contains a myriad of extracellular components including fibrillin, elastin, glycoproteins, and proteoglycans, but is extremely rich in collagen. ${ }^{23}$ Collagen I was found to activate the expression of Rho guanosine triphosphatases (GTPases) and sarcoma (Src) kinases, key regulators of actin cytoskeleton in micro vascular ECs, through the $\beta 1$ integrin. Consequently, ECs cultured on collagen I exhibited precapillary cord like structure development due to disruption of vascular endothelial (VE)-cadherin. ${ }^{24}$ In sharp contrast, Rho GTPases and Src kinases were not activated when ECs were cultured on laminin-1, which prevented the development of precapillary cord like structures by inhibiting the VE-Cadherin disruption. ${ }^{24}$ Several other studies have confirmed the positive influence of collagen on capillary network formation during MSC-EC co-culture. ${ }^{16,19}$ Besides collagen, adipose derived and bone marrow derived stromal cells co-cultured with ECs in fibrin embedded spheroids also developed vascular network like structures. ${ }^{25}$ In order to mimic the native microenvironment more closely, we have developed decellularized ECM from human dermal fibroblast sheets. ${ }^{26}$ Importantly, these ECM sheets inherit the nano-fibrous structure of ECM and support robust vascular network formation during MSC-EC co-culture. ${ }^{26}$ As an alternative for natural ECM components, various biodegradable and biocompatible synthetic polymers can be used to develop scaffolds for co-cultures, such as polycaprolactone (PCL), poly(l-lactic acid) (PLA), poly(glycolic acid) (PGA), and poly(lacticco-glycolic) acid (PLGA). ${ }^{27}$ Although these synthetic materials are able to mimic the physical properties of ECM to some extent, they need to be further functionalized to create a more favorable environment for the cells. Combinations of synthetic polymers with naturally derived ECM can be used to fabricate an appropriate matrix for pre-vascularization approaches.

\section{Current problems and future outlook}

Although significant progress has been made in engineering micro vessels by optimizing MSC-EC co-cultures, challenges remain in 
engineering a mature micro vascular network with an open lumen, integrated vessel wall, and a physiological density and structure. Moreover, cell survival after in vivo implantation is another major concern. Studies have shown that MSCs experience a very low survival rate $(<5 \%)$ after in vivo implantation. ${ }^{28}$ Although MSCs are considered as immune-privileged due to the absence of MHC-II expression, ${ }^{29}$ in vivo testing showed that MSCs upregulate MHC-II expression at the inflammation site and can be recognized by the host immune system..$^{30}$ All of these challenges should be considered when designing MSC therapies for future tissue engineering applications.

\section{Acknowledgements}

This study was supported by the National Institutes of Health (1R15CA202656) and the National Science Foundation (1703570).

\section{Conflict of interest}

The author declares that there is no conflict of interest.

\section{References}

1. Gutierrez-Aranda I, Ramos-Mejia V, Bueno C, et al. Human induced pluripotent stem cells develop teratoma more efficiently and faster than human embryonic stem cells regardless the site of injection. Stem Cells. 2010;28(9):1568-1570.

2. Hentze H, Soong PL, Wang ST, et al. Teratoma formation by human embryonic stem cells: Evaluation of essential parameters for future safety studies. Stem Cell Res. 2009;2(3):198-210.

3. Caplan AI, Dennis JE. Mesenchymal stem cells as trophic mediators. $J$ Cell Biochem. 2006;98(5):1076-1084.

4. Caplan AI. Why are MSCs therapeutic? New data: new insight. J Pathol. 2009;217(2):318-324.

5. Folkman J, Hochberg M. Self-regulation of growth in three dimensions. J Exp Med. 1973;138(4):745-753.

6. Adams RH, Eichmann A. Axon guidance molecules in vascular patterning. Cold Spring Harb Perspect Biol. 2010;2(5):a001875.

7. Carvalho RL, Jonker L, Goumans MJ, et al. Defective paracrine signalling by TGFbeta in yolk sac vasculature of endoglin mutant mice: a paradigm for hereditary haemorrhagic telangiectasia. Development. 2004;131(24):6237-6247.

8. Armulik A, Abramsson A, Betsholtz C. Endothelial/pericyte interactions. Circ Res. 2005;97(6):512-523.

9. Liling Ren, Dongyang Ma, Bin Liu, et al. Preparation of three-dimensional vascularized MSC cell sheet constructs for tissue regeneration. Biomed Res Int. 2014;2014:301279.

10. Kim TH, Kim SH, Leong KW, et al. Nanografted substrata and triculture of human pericytes, fibroblasts, and endothelial cells for studying the effects on angiogenesis. Tissue Eng Part A. 2016;22(7-8):698-706.

11. Bowers SL, Meng CX, Davis MT, et al. Investigating human vascular tube morphogenesis and maturation using endothelial cell-pericyte cocultures and a doxycycline-inducible genetic system in 3D extracellular matrices. Methods Mol Biol. 2015;1189:171-189.

12. Zhang L, Qian Z, Tahtinen M, et al. Prevascularization of natural nanofibrous extracellular matrix for engineering completely biological three-dimensional prevascularized tissues for diverse applications. $J$ Tissue Eng Regen Med. 2018;12(3):e1325-e1336.

13. Hass R, Kasper C, Böhm S, et al. Different populations and sources of human mesenchymal stem cells (MSC): A comparison of adult and neonatal tissue-derived MSC. Cell Commun Signal. 2011;9:12.

14. Stolzing A, Jones E, McGonagle D, et al. Age-related changes in human bone marrow-derived mesenchymal stem cells: consequences for cell therapies. Mech Ageing Dev. 2008;129(3):163-173.

15. Lindroos B, Suuronen R, Miettinen S. The potential of adipose stem cells in regenerative medicine. Stem Cell Rev. 2011;7(2):269-291.

16. Ma J, Yang F, Both SK, et al. In vitro and in vivo angiogenic capacity of BM-MSCs/HUVECs and AT-MSCs/HUVECs cocultures. Biofabrication. 2014;6(1):015005.

17. Yuan W, Zong C, Huang Y, et al. Biological, immunological and regenerative characteristics of placenta-derived mesenchymal stem cell isolated using a time-gradient attachment method. Stem Cell Res. 2012;9(2):110-123.

18. Wang M, Yang Y, Yang D, et al. The immunomodulatory activity of human umbilical cord blood-derived mesenchymal stem cells in vitro. Immunology. 2009;126(2):220-232.

19. McFadden TM, Duffy GP, Allen AB, et al. The delayed addition of human mesenchymal stem cells to pre-formed endothelial cell networks results in functional vascularization of a collagen-glycosaminoglycan scaffold in vivo. Acta Biomater. 2013;9(12):9303-9316.

20. Lei Chen, Qi Xing, Qiyi Zhai, et al. Pre-vascularization enhances therapeutic effects of human mesenchymal stem cell sheets in full thickness skin wound repair. Theranostics. 2017;7(1):117-131.

21. Chow DC, Wenning LA, Miller WM, et al. Modeling pO(2) distributions in the bone marrow hematopoietic compartment. II. Modified Kroghian models. Biophys J. 2001;81(2):685-696.

22. Zhang L, Xing Q, Qian Z, et al. Hypoxia created human mesenchymal stem cell sheet for prevascularized 3d tissue construction. Adv Healthc Mater. 2016;5(3):342-352.

23. Pettersson A, Nagy JA, Brown LF, et al. Heterogeneity of the angiogenic response induced in different normal adult tissues by vascular permeability factor/vascular endothelial growth factor. Lab Invest. 2000;80(1):99-115.

24. Liu Y, Senger DR. Matrix-specific activation of Src and Rho initiates capillary morphogenesis of endothelial cells. Faseb $j$. 2004;18(3):457-468

25. Verseijden F, Posthumus-van Sluijs SJ, Pavljasevic P, et al. Adult human bone marrow- and adipose tissue-derived stromal cells support the formation of prevascular-like structures from endothelial cells in vitro. Tissue Eng Part A. 2010;16(1):101-114.

26. Zhang L, Qian Z, Tahtinen M, et al., Prevascularization of natural nanofibrous extracellular matrix for engineering completely biological three-dimensional prevascularized tissues for diverse applications. $J$ Tissue Eng Regen Med. 2018;12(3):1325-1336.

27. Tanaka M, Kazuhiro Sato, Erika Kitakami, et al. Design of biocompatible and biodegradable polymers based on intermediate water concept. Polymer Journal. 2014;47:114-121.

28. Abraham MR, Gerstenblith G. Preconditioning stem cells for cardiovascular disease: an important step forward. Circ Res. 2007;100(4):447-449.

29. Patel SA, Sherman L, Munoz J, et al. Immunological properties of mesenchymal stem cells and clinical implications. Arch Immunol Ther Exp (Warsz). 2008;56(1):1-8.

30. Matthay MA. Advances and challenges in translating stem cell therapies for clinical diseases. Transl Res. 2010;156(3):107-111. 